\title{
Understanding the potential of climate teleconnections to project future groundwater drought
}

Rust, W.a; Holman, I. ${ }^{\text {a }}$ Bloomfield, J. ${ }^{\text {b }}$ Cuthbert, M. ${ }^{\text {c }}$ Corstanje, R. ${ }^{d}$

a Cranfield Water Science Institute (CWSI), Cranfield University, Bedford MK43 OAL

b British Geological Survey, Wallingford, OX10 8ED

c School of Earth and Ocean Sciences, Cardiff University, Park Place, Cardiff, CF10 3AT

d Centre for Environment and Agricultural Informatics, Cranfield University, Bedford MK43 OAL

\section{Supplementary Material}

Table 1 - Borehole sites used

\begin{tabular}{|c|c|c|c|c|c|c|}
\hline $\begin{array}{l}\text { Site } \\
\text { Number }\end{array}$ & Site ID & Borehole Name & Aquifer Group & $\begin{array}{l}\text { Record } \\
\text { length } \\
\text { (months) }\end{array}$ & $\begin{array}{l}\text { Number } \\
\text { of gaps }\end{array}$ & $\begin{array}{l}\text { Longest gap } \\
\text { (months) }\end{array}$ \\
\hline 1 & SP90/64 & Ashley Green STW & Chalk & 350 & 0 & 0 \\
\hline 2 & SY68/34 & Ashton Farm & Chalk & 516 & 2 & 4 \\
\hline 3 & TA10/63 & Aylesby & Chalk & 460 & 2 & 9 \\
\hline 4 & TQ21/161 & Beeding Hill & Chalk & 447 & 1 & 4 \\
\hline 5 & SU61/47 & Catherington & Chalk & 576 & 2 & 4 \\
\hline 6 & TQ01/133 & Chantry Post Sullington & Chalk & 456 & 5 & 17 \\
\hline 7 & SU81/1 & Chilgrove House & Chalk & 2,173 & 0 & 0 \\
\hline 8 & TQ25/86 & Chipstead & Chalk & 893 & 7 & 10 \\
\hline 9 & SU34/8D & Clanville Lodge Gate & Chalk & 253 & 1 & 4 \\
\hline 10 & SU71/23 & Compton House & Chalk & 1,476 & 2 & 13 \\
\hline 11 & SE94/5 & Dalton Holme & Chalk & 1,537 & 0 & 0 \\
\hline 12 & TM15/112 & Dial Farm & Chalk & 585 & 3 & 5 \\
\hline 13 & TL79/25 & Frying Pan Lodge & Chalk & 545 & 3 & 4 \\
\hline 14 & SU47/141 & Gibbet Cottages & Chalk & 521 & 0 & 0 \\
\hline 15 & TL89/37 & Grimes Graves & Chalk & 449 & 3 & 8 \\
\hline 16 & TA02/104 & Horkstow Road & Chalk & 417 & 1 & 3 \\
\hline 17 & TQ31/46 & Houdean Bottom & Chalk & 481 & 1 & 3 \\
\hline 18 & SU33/12 & Kings Somborne & Chalk & 363 & 1 & 6 \\
\hline 19 & TL12/122 & Lilley Bottom & Chalk & 438 & 0 & 0 \\
\hline 20 & TR14/9 & Little Bucket Farm & Chalk & 553 & 1 & 4 \\
\hline 21 & ST07/10 & Pant y Lladron & Chalk & 262 & 1 & 5 \\
\hline 22 & TL44/12 & Redlands Hall & Chalk & 643 & 2 & 17 \\
\hline 23 & SU17/57 & Rockley & Chalk & 1,018 & 2 & 9 \\
\hline 24 & TL84/6 & Smeetham Hall Cottages & Chalk & 622 & 3 & 11 \\
\hline 25 & TF83/1 & South Creake & Chalk & 749 & 4 & 9 \\
\hline 26 & SU78/45A & Stonor Park & Chalk & 670 & 1 & 3 \\
\hline 27 & TL33/4 & Therfield Rectory & Chalk & 1,610 & 2 & 48 \\
\hline 28 & SU74/40B & Tile Barn Farm & Chalk & 544 & 1 & 121 \\
\hline 29 & SU04/2 & Tilshead & Chalk & 613 & 6 & 23 \\
\hline 30 & TF81/2A & Washpit Farm & Chalk & 802 & 5 & 5 \\
\hline 31 & TQ25/13 & Well House Inn & Chalk & 888 & 5 & 4 \\
\hline 32 & TV59/7C & West Dean No. 3 & Chalk & 922 & 1 & 22 \\
\hline
\end{tabular}




\begin{tabular}{|c|c|c|c|c|c|c|}
\hline 33 & SU01/5B & West Woodyates Manor & Chalk & 901 & 8 & 9 \\
\hline 34 & SE95/6 & Wetwang & Chalk & 544 & 1 & 3 \\
\hline 35 & SK15/16 & Alstonefield & Limestone & 513 & 1 & 5 \\
\hline 36 & SE44/80 & Brick House Farm & Limestone & 448 & 1 & 15 \\
\hline 37 & SE35/4 & Castle Farm & Limestone & 549 & 4 & 25 \\
\hline 38 & SN00/11 & Greenfield Garage & Limestone & 274 & 0 & 0 \\
\hline 39 & SK17/13 & Hucklow South & Limestone & 574 & 5 & 7 \\
\hline 40 & TF03/37 & New Red Lion & Limestone & 932 & 1 & 296 \\
\hline 41 & NZ22/53 & Newton Aycliffe & Limestone & 584 & 5 & 75 \\
\hline 42 & NZ21/29 & Swan House & Limestone & 556 & 1 & 33 \\
\hline 43 & SP00/62 & Ampney Crucis & Oolite & 699 & 0 & 0 \\
\hline 44 & ST88/62A & Didmarton 1 & Oolite & 472 & 4 & 7 \\
\hline 45 & ST51/57 & Over Compton & Oolite & 545 & 6 & 5 \\
\hline 46 & NY00/328 & Brownbank Layby & Sandstone & 487 & 5 & 26 \\
\hline 47 & SX99/37B & Bussels No. 7A & Sandstone & 544 & 0 & 0 \\
\hline 48 & SD27/6B & Furness Abbey & Sandstone & 526 & 4 & 21 \\
\hline 49 & SJ62/112 & Heathlanes & Sandstone & 547 & 4 & 7 \\
\hline 50 & SJ15/13 & Llanfair Dyffryn Clwyd & Sandstone & 540 & 0 & 0 \\
\hline 51 & SK67/17 & Morris Dancers & Sandstone & 568 & 1 & 9 \\
\hline 52 & NX97/2 & Newbridge & Sandstone & 287 & 0 & 0 \\
\hline 53 & SK00/41 & Nuttalls Farm & Sandstone & 510 & 1 & 8 \\
\hline 54 & NT94/3B & Royalty Observation & Sandstone & 314 & 1 & 3 \\
\hline 55 & NY63/2 & Skirwith & Sandstone & 460 & 2 & 35 \\
\hline 56 & SK10/9 & Weeford Flats & Sandstone & 603 & 2 & 12 \\
\hline 57 & SD41/32 & Yew Tree Farm & Sandstone & 525 & 4 & 27 \\
\hline 58 & ST30/7 & Lime Kiln Way & Greensand & 578 & 2 & 8 \\
\hline 59 & TQ41/82 & Lower Barn Cottage & Greensand & 502 & 3 & 3 \\
\hline
\end{tabular}




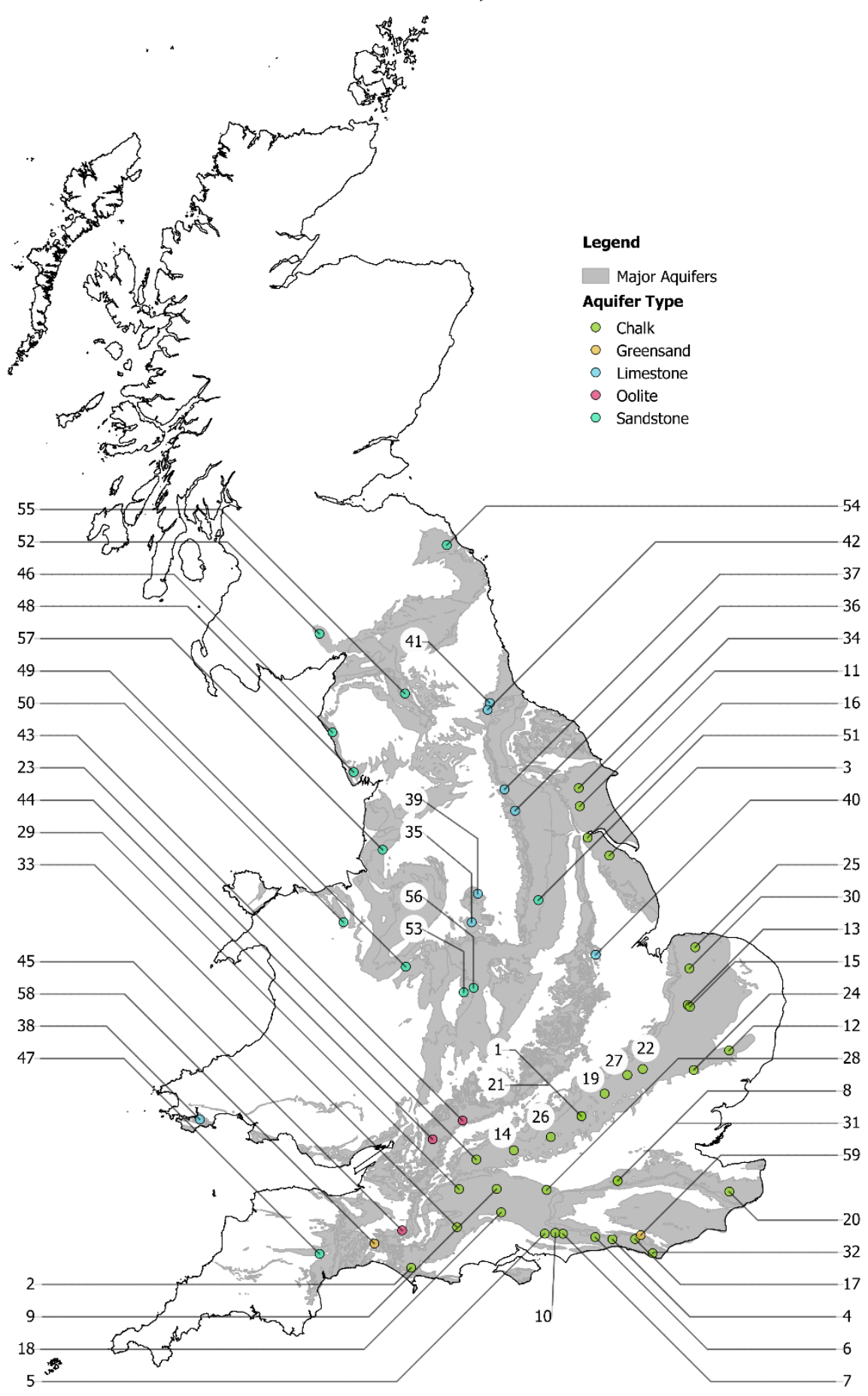

Figure 1 - Location of observation boreholes used in this study with their Site numbers, and major aquifers in the UK 

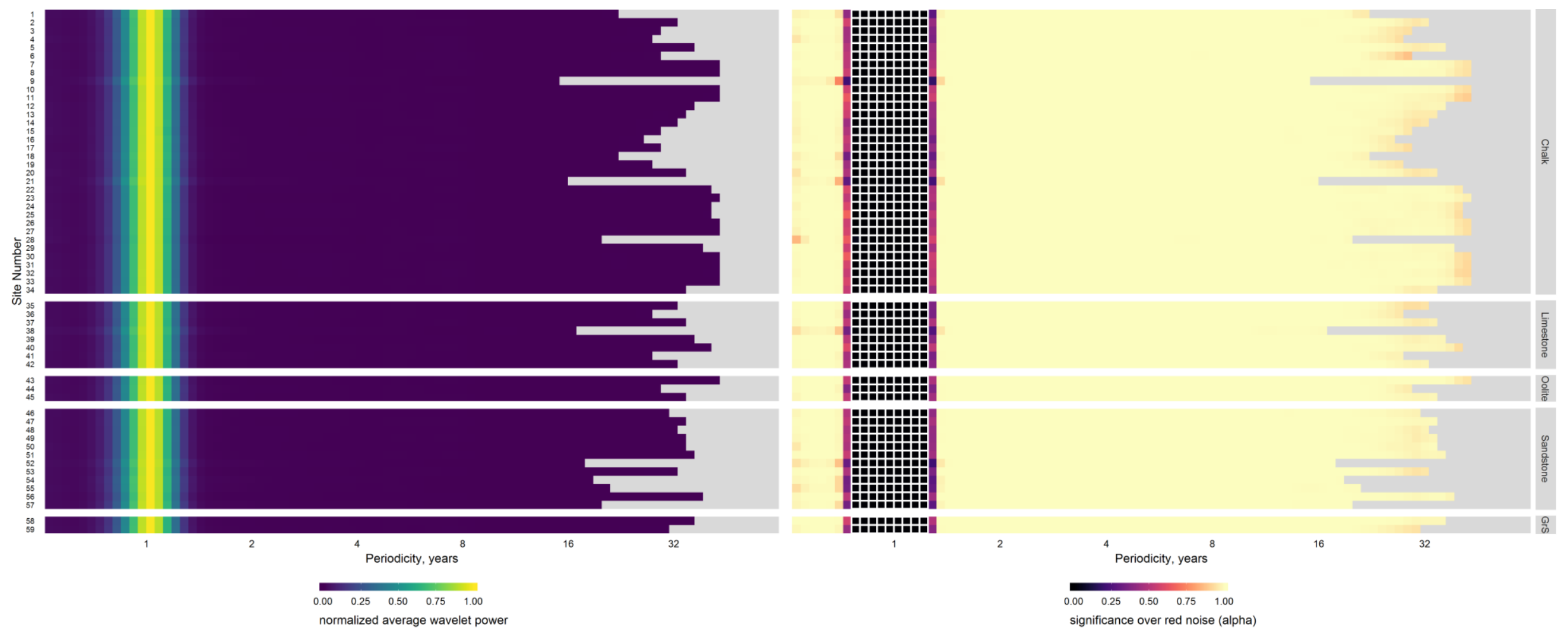

Figure 3 - Normalised average wavelet power spectra (left) and wavelet power significance alphas (right) for monthly PET time series for co-locations of the 59 index boreholes. In the right-hand figure, boxes outlined in white are those powers that are significant over red noise to a 95\% confidence interval ( $a<=0.05)$. 